\title{
Flushing Disorders Associated with Gastrointestinal Symptoms: Part I, Neuroendocrine Tumors, Mast Cell Disorders and Hyperbasophila
}

\author{
Vaibhav Rastogi, MD; Devina Singh, MD; Joseph J. Mazza, MD; \\ Dipendra Parajuli, MD, and Steven H. Yale, MD
}

\begin{abstract}
Flushing is the subjective sensation of warmth accompanied by visible cutaneous erythema occurring throughout the body with a predilection for the face, neck, pinnae, and upper trunk where the skin is thinnest and cutaneous vessels are superficially located and in greatest numbers. Flushing can be present in either a wet or dry form depending upon whether neural-mediated mechanisms are involved. Activation of the sympathetic nervous system results in wet flushing, accompanied by diaphoresis, due to concomitant stimulation of eccrine sweat glands. Wet flushing is caused by certain medications, panic disorder and paroxysmal extreme pain disorder (PEPD). Vasodilator mediated flushing due to the formation and release of a variety of biogenic amines, neuropeptides and phospholipid mediators such as histamine, serotonin and prostaglandins, respectively, typically presents as dry flushing where sweating is characteristically absent. Flushing occurring with neuroendocrine tumors accompanied by gastrointestinal symptoms is generally of the dry flushing variant, which may be an important clinical clue to the differential diagnosis. A number of primary diseases of the gastrointestinal tract cause flushing, and conversely extra-intestinal conditions are associated with flushing and gastrointestinal symptoms. Gastrointestinal findings vary and include one or more of the following non-specific symptoms such as abdominal pain, nausea, vomiting, diarrhea or constipation. The purpose of this review is to provide a focused comprehensive discussion on the presentation, pathophysiology, diagnostic evaluation and management of those diseases that arise from the gastrointestinal tract or other site that may cause gastrointestinal symptoms secondarily accompanied by flushing. This review is divided into two parts given the scope of conditions that cause flushing and affect the gastrointestinal tract: Part I covers neuroendocrine tumors (carcinoid, pheochromocytomas, vasoactive intestinal polypeptide, medullary carcinoma of the thyroid), polyneuropathy, organomegaly, endocrinopathy, monoclonal protein, skin changes (POEMS), and conditions involving mast cells and basophils; while Part 2 covers dumping syndrome, mesenteric traction syndrome, rosacea, hyperthyroidism and thyroid storm, anaphylaxis, panic disorders, paroxysmal extreme pain disorder, and food, alcohol and medications.
\end{abstract}

Keywords: Flushing; Neuroendocrine Tumor; Systemic Mastocytosis;

Gastrointestinal; human

$\mathrm{V}$ ascular tone, under normal physiologic conditions is regulated by the autonomic nervous system. Alteration in the neurologic control of the cutaneous smooth muscle vasculature is responsible for flushing. ${ }^{1}$ Flushing is an intermittent or persistent subjective sensation of warmth accompanied by visible skin erythema occurring throughout the body with a predilection for the face, neck, pinnae and upper trunk, with the face most commonly involved. ${ }^{1,2}$ It is at these locations where the vessel diameter is wider, vascular response is greater and cutaneous blood vessels have greater capacitance and numbers per unit volume and superficially located. ${ }^{1,3}$ Increased blood flow
Corresponding Author: Steven H.Yale MD, University of Central Florida College of Medicine, HCA Consortium Graduate Medical Education, North Florida Regional Medical Center, 6500 W Newberry Rd, Gainesville, FL 32605, Tel: (7I5) 383-0928

Email: steven.yale.md@gmail.com
Received: July 31, 2017

Revised: November 30, 2017

Accepted: December 21, 2017

doi:10.3121/cmr.2017.1379a 
Table 1. Flushing Mechanisms

\author{
I. Autonomic Neural Mediated (Wet Flushing) \\ Panic disorder \\ Paroxysmal extreme pain syndrome \\ Medications (see Table 6 in Part 2) \\ Poisoning: \\ Organophosphate insecticides
}

\title{
II. Direct Vasodilator Mediated (Dry Flushing)
}

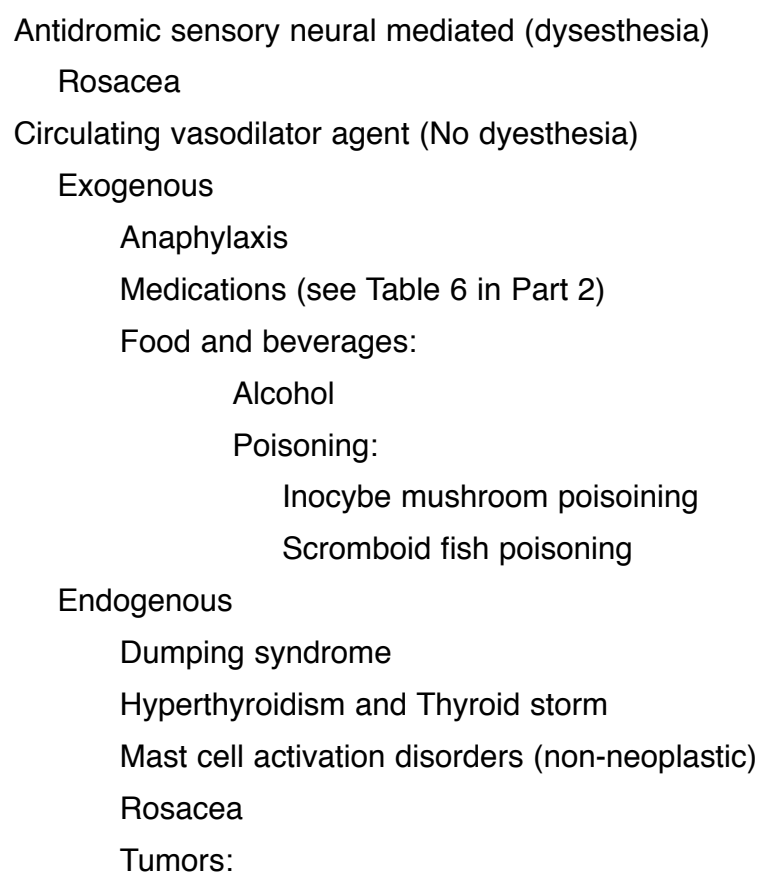

\author{
Ganglioneuromas \\ Medullary carcinoma of the thyroid \\ Neuroendocrine tumors \\ Pheochromocytoma \\ Pancreatic tumors (VIPoma, Neurotensinoma) \\ POEMS syndrome \\ Systemic mastocytosis
}

through the skin under physiologic (blushing) and pathologic conditions results in cutaneous warmth and blood vessel enlargement caused by endogenous vasodilatory mediators (eg, histamine, substance $\mathrm{P}$, serotonin and prostaglandins), exogenous agents (eg, medications) or autonomic neuralmediated mechanisms. ${ }^{2}$

When dysregulation of neurologic control results in flushing, it is of the wet variant (accompanied by sweating) as sympathetic autonomic nerves also innervate eccrine sweat glands. Example causes of wet flushing are medications, panic disorders, and paroxysmal extreme pain syndrome (Table 1). In this scenario, diaphoresis occurs at the same time as erythema. Diaphoresis occurring after flushing is a marker for severity and suggests the presence of concomitant hypotension and secondary activation of sympathetic mediated reflexes. $^{4}$
Vasodilator mediated flushing, as opposed to neurogenic flushing, is usually of the dry flushing variety since sweating does not occur. It is more commonly found in patients with endogenous pathologic conditions such as solid tumors (eg, carcinoid, vasoactive intestinal peptide tumors (VIPomas), or exogenous factors caused by food, alcohol, and medications (Table 1). Flushing can be spontaneous, episodic, invoked (eg, by diagnostic studies, emotional disturbances, and temperature changes), or persistent and may be accompanied by pain or burning sensation in the case of antidromic sensory neural-mediated mechanism (eg, mast cell activation disorder and rosacea).,

A comprehensive history and physical examination should be obtained inquiring about frequency and factors, if any, that precipitates the flushing episode, color and duration of the 
Flushing with GI symptoms

- Review for food, alcohol, medication, poisoning, occupation and if present remove exposure.

- Hyperacute onset with angioedeman evaluate for anaplylaxis.

- If neither of these findings are present evaluate based on the following symptoms:

-Headache, tachycardia, sweating, hypertension $\rightarrow$ pheochromocytomas, ganglioneuroma

•Bronchospasm. gastrointestinal bleeding. obstruction, vasoconstriction $\rightarrow$ NETs

-Vasodilation, hypotension, tachycardia $\rightarrow$ Mast cell activation disorders

•Polyneuropathy, lymphadenopathy, endocrine symptoms $\rightarrow$ POEMS Syndrome

•Hypokalemia, achlorhydria $\rightarrow$ VIPoma

•Palpitation., tremor, fatigue, hair thinning $\rightarrow$ Hyperthyroidism

-Post gastric by-pass/ bariatric surgery with post-prandial rapid gastric emptying $\rightarrow$ Dumping syndrome

-Recent abdominal surgery $\rightarrow$ Mesenteric traction syndrome

-Dysphagia, dyspnea $\rightarrow$ Medullary carcinoma of thyroid

-Visceral pain paroxysm with family history $\rightarrow$ Paroxysmal extreme pain disorder

- Behavioral/ emotional/ mood disorders $\rightarrow$ Panic disorder

-Dysesthesia and cutaneous findings $\rightarrow$ Rosacea and other dermatological disorders

•Basophilic count $>1000$ per ul blood $\rightarrow$ Hyperbasophic disorders

\section{Flushing without GI symptoms}

- Menopause

- Medications (e.g. GnRH)

- Physiological (heat, anger, embarrassment, drinks)

- Auriculotemporal syndrome (Frey's Syndrome)

- Harlequin syndrome

- Pseudocarcinoid syndrome in secondary male hypogonadism

- Spinal cord injury above T6 (autonomic hyperreflexia)

- Rapid increase in intracranial pressure

- Migraine headaches

- Horseshoe kidney (Rovsing`s syndrome)

- Renal cell carcinoma

Figure 1. Diagnostic approach to flushing with and without gastrointestinal symptoms.

flush, presence of cutaneous skin lesions and pruritus, whether diaphoresis is present and if it occurs prior to or follows flushing, and if pain or burning is present. All medications including prescription, over the counter, and herbals should be reviewed. An underlying systemic cause of flushing should be considered if flushing episodes occur with other symptoms such as headache, syncope, diarrhea, and abdominal pain (Figure 1).

Primary and secondary gastrointestinal diseases associated with flushing can be classified based on the pathophysiologic mechanism of flushing as autonomic neural-mediated, or direct vasodilator-mediated with vasodilator-mediated responses occurring directly through activation of antidromic sensory nerves, or indirectly from endogenous or exogenous vasodilator agents (Table 1).

Gastrointestinal symptoms occurring with flushing are nonspecific and include nausea, vomiting, diarrhea and abdominal pain. In the case of vasodilatory flushing, symptoms result from the release of a variety of mediators such as histamine, serotonin, calcitonin, and vasoactive intestinal peptide (VIP) that potentiate secretion through variations in sodium transport across the intestinal lining. ${ }^{1}$ Alteration in the absorption of sodium via calcium, cyclic adenosine monophosphate (cAMP) and cyclic guanosine monophosphate (cGMP) mechanisms results in diarrhea. ${ }^{5}$

The aim of this overview is to summarize the clinical presentation, pathophysiology, diagnostic evaluation and management of those diseases that primarily or secondarily affect the gastrointestinal tract and cause flushing. Hence, other conditions such as menopausal flushing, physiological flushing, and drugs which cause flushing without gastrointestinal involvement, as part of the course of their condition will not be covered in this review. We intended this paper to be a concise discussion on common and rare causes that provides a novel framework from which clinicians may find helpful when approaching patients with flushing and gastrointestinal symptoms. Given the scope of information, the paper is divided into two parts: in Part 1 we cover neuroendocrine tumors, polyneuropathy, organomegaly, endocrinopathy, monoclonal protein, skin changes (POEMS), mast cell and basophilic disorders, and in Part 2 we cover dumping syndrome, rosacea, mesenteric tractions syndrome, hyperthyroidism and thyrotoxicosis, panic disorders, anaphylaxis, paroxysmal extreme pain syndrome, food, alcohol and medication.

\section{Methods}

MEDLINE and Cochrane Library databases were searched using individual and the MeSH terms flushing, flushing disorders, carcinoid tumors, gastrointestinal, neuroendocrine tumors, mast cell disorders, drugs, alcohol, anaphylaxis, allergy, dumping syndrome, mesenteric traction syndrome, and rosacea. "Flushing disorders" alone yielded 2080 manuscripts and "flushing disorders and gastrointestinal" resulted in 118 manuscripts. Key words related to each MeSH term in the tile and abstract were also queried. The search was limited to human clinical studies in the English literature published prior to April 1, 2017. We reviewed bibliographies of retrieved studies as well as reviews for additional relevant 
studies. Three reviewers independently screened titles, abstracts and full text of potentially eligible articles to identify studies meeting inclusion criteria. Disagreement in inclusion was resolved by consensus. Information was obtained primarily from observational cohort studies, systematic reviews, case series, controlled clinical trials, and consensus or position societal statements. To the extent possible, we limited our selection of manuscripts to those published in the past 10 years. We restricted the content to causes, clinical presentation and gastrointestinal involvement, and diagnosis and treatment of flushing disorders and conditions associated with gastrointestinal symptoms.

\section{Neuroendocrine Tumors}

Neuroendocrine tumors (NETs), previously classified as carcinoid tumors, arise from enterochromaffin or Kluchitsky cells and occur throughout the gastrointestinal tract, most commonly in the mid-gut (duodenum, small intestines, cecum, vermiform appendix, ascending colon and right two thirds of transverse colon), as well as the lungs, thymus and rarely the kidneys and ovaries. ${ }^{6,7}$ NETs constitute $35 \%$ to $42 \%$ of all tumors of the small bowel and are the primary type located in the ileum and appendix with $26 \%$ to $30 \%$ being multicentric throughout the bowel. ${ }^{8-10}$ Appendiceal NETs are the most common type of tumors and account for greater than $50 \%$ of the primary tumors of the appendix. ${ }^{11}$ Appendiceal NET's are more likely to have a benign clinical course because of their early presentation and detection. ${ }^{8} \mathrm{~A}$ rising incidence of NETs from the period of 2004 of 1.09 per 100,000 to $5.25 / 100,000$ in 2004 presumably reflects early detection due to the use of advanced diagnostic imaging and endoscopic techniques..$^{1,12}$

NETs of the gastrointestinal tract are classified by the World Health Organization (WHO) into four groups (Grade 1 NET, Grade 2 NET, neuroendocrine carcinoma (large or small cell types) and mixed adenoneuroendocrine carcinoma based on the tumors mitotic counts and $\mathrm{Ki}-67$ proliferation index parameters that assess the degree of differentiation on histopathology assessment. ${ }^{13}$ Further subtyping of gastrointestinal NET and their metastasis is based on their location and the predominant, if any, hormone, biogenic amine, or vasoactive substances secreted.

The majority of NETs is asymptomatic and identified incidentally. Flushing, a well-recognized and described complication, occurs in only $10 \%$ of patients with NET. ${ }^{7}$ Thus, symptoms when present signify metastases unless local symptoms, such as obstruction, bleeding or ischemia caused by the tumor, occur earlier in the disease course. ${ }^{6}$ There is an extensive variation and unpredictable clinical course of disease progression with an average reported five-year survival ranging from $14 \%$ to $54 \%$ in patients with metastatic disease. $^{14}$

\section{Clinical Presentation and Gastrointestinal Involvement}

The term carcinoid syndrome is reserved to describe the constellation of intermittent or persistent symptoms of cutaneous flushing, diarrhea, cardiac valvular lesions and bronchospasm related to excessive serotonin production. ${ }^{6}$ These symptoms often appear prior to the signs or symptoms of the primary tumor. Gastrointestinal NETs that have not metastasized typically do not cause carcinoid syndrome since the liver metabolizes serotonin, the primary mediator released from these tumors. Thus, patients with more advanced disease develop carcinoid syndrome since hepatic metastases bypass liver metabolism of serotonin that is delivered directly into the systemic circulation. ${ }^{7}$ The syndrome however may rarely occur in patients with disease involving the retroperitoneum, such as in rectal NETs, since venous drainage bypasses the liver directly into the systemic paravertebral veins or other anomalous venous routes. ${ }^{15,16}$

Carcinoid syndrome commonly occurs with midgut tumors since it is this region of the bowel where serotonin concentration is highest. Foregut (gastric and duodenal) and hindgut (rectal) tumors rarely cause an atypical carcinoid syndrome characterized by flushing, hypotension, edema, and bronchoconstriction caused by excessive production of 5-hydroxytryptamine (5-HTP) due to the absence of the enzyme aromatic acid decarboxylase within tumors in these locations which converts 5-HTP to 5-HT (serotonin). ${ }^{7}$

Cutaneous dry flushing, found in more than $85 \%$ of patients, is the hallmark of carcinoid syndrome. ${ }^{13}$ Hypotension with sweating may occur in patients with significant volume loss caused by diarrhea in carcinoid syndrome. However, this is a secondary phenomenon that occurs in response to hypovolemia and hypotension from diarrhea. Flushing, mediated by the release of vasoactive substances 5-HT, tachykinins and bradykinins occurs in paroxysms, provoked by external factors such as alcohol, spices, stress, and epinephrine and lasts up to 30 minutes. ${ }^{6}$ Neither aspirin nor exercise precipitates flushing. It should be recognized that serotonin alone is not the only humoral mediator responsible for flushing since it occurs in patients with low to normal 5-hydroxyindoleacetic acid (5-HIAA) levels. ${ }^{17}$

The distinguishing features of the flush may provide a clinical clue to the location of the NET: foregut NET flush is reddish brown in color, ${ }^{18}$ midgut NETs flushing occurs briefly and is dark red in color. A purplish to violet hue caused by large cutaneous blood vessels containing deoxygenated blood, with telangiectasia may occur in longstanding midgut tumors. ${ }^{2,19}$ Hindgut colon NETs, similar to other neoplasms occurring in this region, present nonspecifically with hematochezia and a change in bowel patterns with flushing and diarrhea characteristically absent even with metastatic disease. ${ }^{20}$ In contrast, a bright salmon confluent pink to red flush is found in bronchial tumors. Additionally, bronchial carcinoid syndrome flushing tends to be more protracted lasting hours to days, occurs in the absence of triggering foods, and may be associated with other clinical features including chemosis, facial edema, hypotension and oliguria and telangiectasias. ${ }^{18,21}$ 
Serotonin is believed to be responsible for increased gastrointestinal transit time as well as stimulating fluids and electrolytes secretion within the bowel through activation of 5-HT2A receptors. ${ }^{22}$ Approximately $80 \%$ of patients complain of non-bloody watery diarrhea and abdominal cramping typically associated with food consumption ${ }^{22-24}$ not necessarily occurring concomitantly with the flushing episodes. ${ }^{23,25}$ Abdominal pain and cramping may be due to diarrhea, intestinal obstruction or mesenteric fibrosis. ${ }^{22}$ Mesenteric fibrosis is an important complication occurring in $66 \%$ of patients with midgut carcinoid tumors since it may result in mesenteric shortening, kinking, ischemia, volvulus and intestinal obstruction. ${ }^{26-29}$ Bowel ischemia may also be secondary to mesenteric angiopathy and vascular fibroelastosis. ${ }^{26,30}$ Right-heart fibrosis causes right-heart failure and valvular abnormalities potentially leading to a congestive hepatopathy. Elevated levels of 5-hydroxytrpytamin, TGF- $\beta$, tachykinins, neurokinin A, substance $\mathrm{P}$, platelet derived growth factor, connective tissue growth factor (CTGF) and fibroblast growth factor (FGF) have been implicated in causing stromal proliferation and fibrosis at these sites. ${ }^{31,32}$

\section{Diagnosis and Treatment}

Laboratory testing for carcinoid syndrome involves obtaining a 24-hour measurement of urinary excretion of the serotonin metabolite 5-HIAA and plasma/serum chromogranin A $(\mathrm{CgA})$, and in some cases, platelet serotonin, when urinary 5-HIAA results are equivocal. ${ }^{23}$ A sensitivity and specificity of $70 \%$ to $100 \%$ has been reported for chromogranin A for detecting NET. ${ }^{33}$ Urinary 5-HIAA concentration has a reported sensitivity and specificity of $90 \%$. $^{7}$

Diagnostic radiological imaging is required for tumor localization, to determine the extent, disease status and treatment response and follow-up for surveillance after complete resection. ${ }^{34}$ Imaging involves one or a combination of modalities including computed tomography (CT), magnetic resonance imaging (MRI), endoscopic ultrasonography (EUS), and radiolabeled somatostatin analogue scintigraphy using ${ }^{99}$ Tc-labeled or ${ }^{111}$ In-pentetreoide ( ${ }^{111}$ In-DTPApentetreotide; Octreoscan) and in those negative on Octreoscan, ${ }^{123}$ Iodine-metaiodobenzylguanidine ( ${ }^{123}$ I-MIBG) scintigraphy.

Expression and identification of somatostatin receptors subtype 2A (SST-2A) is particularly useful in guiding imaging and predicting response to treatment with somatostatin analogues or peptide-receptor radionuclide therapy (PRRT). ${ }^{35}$ Positron emission tomography-computed tomography with ${ }^{18} \mathrm{~F}$-flurodeoxyglucose (FDG) imaging (PET-CT) is reserved for patients with undifferentiated and nonsecretory tumors due to their increased proliferative activity (Ki-67 index) or in patients with negative or equivocal imaging on ${ }^{123} \mathrm{I}-\mathrm{MIBG} .{ }^{7,33}$ Endoscopic or percutaneous obtained biopsy is required for histopathology to confirm the diagnosis.
Treatment may include, based on the stage of the disease, one or more of the following modalities including surgical resection, cytoreductive therapy, chemotherapy, somatostatin analogues, and targeted therapies. ${ }^{34}$ Cytoreductive therapy has been advocated for treatment of early stage disease as well as hepatic and peritoneal metastases. ${ }^{7}$ For hormonally active tumors, patients should receive pre-operative as well as intraoperative somatostatin analogues, octreotide or lanreotide, to prevent carcinoid crisis that may occur during anesthesia and tumor manipulation. ${ }^{7}$ Hepatic metastases that are not amenable to cytoreduction therapy may potentially benefit from hepatic artery embolization therapies including bland embolization, chemoembolization and radioembolization. ${ }^{7}$ Liver transplantation can be considered when metastases are limited to the liver and there is no evidence for disease progression in the past one year. ${ }^{34}$ There is limited but emerging experience pertaining to the use of targeted nuclear therapies including ${ }^{123}$ I-meta-iodobenzylguanidine (MIBG) and radiolabeled somatostatin analogues. ${ }^{7}$ Systemic therapies including interferon-alpha and everolimus are an option for cases refractory to somatostatin analogues. ${ }^{34}$ For those patients for whom surgical resection is not an option, symptoms, and hormone overproduction or paraneoplastic syndromes are managed with octreotide or lanreotide. Significant improvements in treatment strategies, as well as the use of somatostatin analogues based on its antiproliferative properties in slowing tumor growth and control of symptoms of flushing and diarrhea has resulted in decreased mortality, morbidity and improved quality of life. ${ }^{34-37}$

\section{Pheochromocytoma}

Pheochromocytomas are catecholamine-secreting tumors derived from the chromaffin cells of the sympathoadrenal system. Those arising from sympathetic and parasympathetic preganglia cells are referred to as paragangliomas. Paragangliomas unlike pheochromocytomas most commonly occur in the glomus jugulare or carotid bodies, are more likely to be malignant, and usually do not produce systemic symptoms secondary to catecholamine secretion. ${ }^{38}$

Pheochromocytomas are rare tumors with an annual incidence of 2 million to 8 million per year. The majority of patients presenting during their fourth or fifth decade with males and females equally affected. ${ }^{39}$ Paragangliomas are more commonly found in males with a median age of diagnosis of 43 years. $^{40}$ Approximately $30 \%$ of patients have a familial disorders such as von-Hippel-Lindau syndrome or multiple endocrine neoplasia type 2 (MEN Type II). ${ }^{41}$ Patients suspected of having a familial disease based on clinical findings or family history should undergo genetic testing to ascertain if genetic mutations are present since these patients require lifelong surveillance as they have a higher probability for developing other malignancies. ${ }^{41}$

\section{Clinical Presentation with Gastrointestinal Involvement}

The classic triad, present in only half of patients, consists of sporadic, episodic, or paroxysms symptoms of headache, 
sweating and tachycardia lasting 10 minutes to 60 minutes. Hypertension is a common clinical finding and is sustained or paroxysmal in half of the cases. ${ }^{39}$ Other clinical manifestations include anxiety, flushing, pallor, Raynaud's phenomenon, tremors, chest pain, dizziness, urinary symptoms including polyuria and nocturia, nausea, vomiting, diarrhea, constipation and weight loss. The release of catecholamines norepinephrine and epinephrine is responsible for these symptoms. ${ }^{39,42}$ Pallor due to cutaneous vasoconstriction rather than flushing more commonly occurs during a spell due to the presence of elevated norepinephrine levels. ${ }^{43}$ Flushing following an acute episode is mediated by dopamine, epinephrine, vasoactive intestinal polypeptide, calcitonin gene-related peptide (CGRP) and adrenomedullin. ${ }^{19,44,45}$

Rarely, pheochromocytomas are reported to causes watery diarrhea, hypokalemia and achlorhydria (WDHA) syndrome due to the release of vasoactive intestinal polypeptide (VIP). ${ }^{46}$ Extra-adrenal pheochromocytomas are associated with increased risk for developing gastrointestinal bleeding. ${ }^{42}$

\section{Diagnosis and Treatment}

Measurement of fractionated metanephrine and catecholamines in a 24-hour urine and free plasma metanephrine are two important initial screening biochemical tests, with specific selection based on the clinical probability of disease. When clinical suspicion is low, a 24-hour urinary fractionated catecholamines should be ordered as the initial screening test and fractionated metanephrine levels added when there is heightened clinical suspicion of disease. ${ }^{41}$ Diagnostic imaging should be restricted to patients whose metanephrine and catecholamine values are at least two-fold higher from baseline. Computed tomography (CT) or magnetic resonance imaging (MRI) of the abdomen is initially used to identify the tumor with ${ }^{123}$ I-metaiodobenzylguanidine (MIBG) scintigraphy and fludeoxyglucose-positron emission tomography (FDGPET) to confirm the diagnosis in cases of diagnostic uncertainty. ${ }^{41}$

Surgical adrenalectomy is the primary treatment for resectable pheochromocytomas resulting in symptom resolution and favorable short and long-term clinical outcome and tumor recurrence rate is less than $10 \%$ for patients with benign disease. ${ }^{39,47}$ Preoperative hypertension is managed using alpha-adrenergic blockers for 10 to 14 days followed by a beta-blocker for 2 to 3 days.

\section{Vasoactive Intestinal Polypeptide Tumors (VIPoma)}

NETs of the pancreas produce local hormones of the islet cells such as insulin, glucagon, somatostatin and pancreatic polypeptide and in some cases ectopic hormones including calcitonin, adreno-corticotopin (ACTH), neurotensin or vasoactive intestinal polypeptide (VIP). Vasoactive intestinal polypeptide tumor (VIPoma) is a rare neuroendocrine tumor with an incidence of 1 in 10 million per year. These tumors occur in patients between the third and fifth decade. ${ }^{48}$ The tumor is located in the tail of the pancreas in $75 \%$ of cases. ${ }^{48}$

\section{Clinical Presentation and Gastrointestinal Involvement}

The syndrome of watery diarrhea, hypokalemia, and achlorhydria occurring in these tumors is referred to by the acronym WDHA. The most prominent and profound symptom of VIPoma's is secretory diarrhea resulting in fluid and electrolyte loss and hence is commonly referred to as pancreatic cholera. Fluids and electrolytes loss may result in various complications including hypotensive shock and thus replacement is the initial step in management. ${ }^{49}$ Laboratory findings may also include hyperchloremic metabolic acidosis or metabolic alkalosis in patients with milder disease. ${ }^{48}$

VIP is structurally homologous to secretin, glucagon and histidine, which accounts for the secretory diarrhea and electrolyte abnormalities. ${ }^{49}$ Other rare neoplasms arising within the abdomen that release VIP and causing flushing and diarrhea, include pheochromocytomas, malignant histiocytoma, neuroblastoma, and ganglioneuroma. ${ }^{2,48}$ These tumors also secrete other polypeptides including pancreatic polypeptide and neurotensin, the latter producing symptoms of edema, hypotension, flushing and the WDHA syndrome; symptoms clinically indistinguishable from a primary VIPoma and are referred to as a neurotensinoma. ${ }^{50,51}$

\section{Diagnosis and Treatment}

Laboratory testing requires measuring serum concentrations for VIP and pancreatic polypeptide, and the chromogranin A radioimmunoassay. Diagnostic imaging with ultrasound, computed tomography and magnetic resonance imaging assists in tumor localization. Functional PET imaging such as ${ }^{68} \mathrm{Ga}$-DOTA fusion PET/CT scans is the most sensitive modality for localizing VIPomas. ${ }^{48}$ Somatostatin receptor scintigraphy is used specifically for tumors that express somatostatin receptors for VIPomas located at extrapancreatic sites. $^{49}$

Surgical resection is the primary treatment and there is no widespread consensus about the use of adjuvant chemotherapy post-surgery. Octreotide, interferon-alpha and glucocorticoids have been prescribed. ${ }^{48}$

\section{Medullary Carcinoma of Thyroid Gland}

Medullary carcinoma of thyroid (MCT) is a rare tumor arising from C-cells, or parafollicular cells, of thyroid gland. MCT accounts for $3 \%$ to $10 \%$ of the thyroid carcinomas in United States. ${ }^{52,53}$ Although these tumors most commonly occur sporadically, hereditary forms, part of multiple endocrine neoplasia (MEN) syndromes types $2 \mathrm{~A}$ and $2 \mathrm{~B}$, arise due to a germ-line mutation in the RET proto-oncogene. ${ }^{53}$

\section{Clinical Presentation and Gastrointestinal Involvement}

The mean age of presentation is 38 years. ${ }^{53}$ Symptoms can be localized or systemic with local symptoms including dysphagia and dyspnea caused by tumor-induced compression of 
Table 2. Primary, Secondary and Idiopathic Classification of Mast Cell Activation Disorders.

Primary

1. Cutaneous Mastocytosis (WHO Classification 2016)

Maculopapular cutaneous mastocytosis (MCM) [urticarial mastocytosis]

Diffuse cutaneous mastocytosis (DCM)

Mastocytoma of skin (cutaneous mastocytoma)

2. Systemic Mastocytosis (WHO Classification 2016)

Smoldering Systemic Mastocytosis (SSM)

Aggressive Systemic Mastocytosis (ASM)

[untransformed and transformation to MCL-ASM-t subvariants]

Indolent Systemic Mastocytosis (ISM)

Systemic mastocytosis with an Associated Hematologic Neoplasm (SM with AHN)

Mast Cell Leukemia (MCL)

[Acute Mast Cell Leukemia (AML) and Chronic Mast Cell Leukemia (CML) subvariants]

\section{Mast cell sarcoma}

Monoclonal Mast cell Activation (MMCA)

\section{Secondary ${ }^{\star *}$}

IgE-mediated hypersensitivity reaction

Drugs

Mast cell hyperplasia (associated with chronic infections, neoplasia, and autoimmune conditions).

Idiopathic $^{\star *}$

Anaphylaxis

Non-Clonal Mast cell Activation Disorder
Systemic mastocytosis*

May be present

May be present

May be present

Cutaneous mastocytosis

May be Present

Generally Absent

May be Present

Generally Absent

Rare

Absent

Absent

Systemic symptoms ${ }^{\star *}$

Present

Present

Present*

Systemic symptoms ${ }^{\star *}$

Present

Present

${ }^{*}$ Flushing, hypotension, pruritus, abdominal pain and diarrhea common.

${ }^{* *}$ Pruritus, flushing, urticaria, angioedema, and dermatographism. Does not include cutaneous mastocytosis skin disorders.

neighboring structures. ${ }^{2}$ Systemic manifestations include face and upper extremity flushing, diarrhea and bone pain attributed to secretion of the neuropeptides histamine, substance $\mathrm{P}$, adrenocorticotropic hormone, ketacalcin, levodopa, calcitonin gene-related polypeptide (CGRP) and prostaglandins. ${ }^{1,2,18}$ Cutaneous flushing is found in $10 \%$ of patients in association with other symptoms such as diarrhea, hoarseness and dysphagia. ${ }^{53,54}$ Diarrhea occurs in two-thirds of patients with metastatic disease, due to the hypersecretory state and/or increased bowel motility induced by neuropeptides. ${ }^{52}$

\section{Diagnosis and Treatment}

Genetic screening for the RET germ line point mutation is a useful method for identifying patients at risk for developing MTC. Radioimmunoassay testing for calcitonin is a highly sensitive method for detecting persistent or recurrent disease. ${ }^{53}$ Fine needle cytology biopsy often confirms the diagnosis. Surgery is the primary treatment for MTC with the approach based on the size and location of the primary tumor, extent of the lymphadenopathy, presence of persistent or recurrent disease, or carrier of germ line RET proto-oncogene. ${ }^{53}$ Diarrhea and flushing resolves with tumor resection with recurrence of symptoms a marker for possible disease relapse. Somatostatins analogous have been shown to be effective in symptomatic patients with metastatic disease. ${ }^{53,55}$ Tyrosine kinase inhibitors are used in the treatment for more advanced disease. Calcitonin doubling time assists in ascertaining disease progression with doubling time of 6 to 12 months correlating with poor prognosis. ${ }^{52}$

\section{POEMS Syndrome}

POEMS, the acronym that describes the clinical manifestation of the syndrome of polyneuropathy, organomegaly, endocrinopathy, monoclonal protein, skin changes (POEMS) is a rare monoclonal plasma cell disorder with paraneoplastic systemic manifestations. ${ }^{56}$

\section{Clinical Presentation and Gastrointestinal Involvement}

Patients generally present during their fifth and sixth decade with one or more symptoms that may including polyneuropathy, organomegaly (hepatomegaly, splenomegaly, lymphadenopathy), endocrinopathy, diarrhea and symptoms of volume overload (edema, ascites and effusion). ${ }^{57}$ Cutaneous findings include flushing, hyperpigmentation, hypertrichosis, plethora and acrocyanosis of the skin are attributed to elevated vascular endothelial growth factor (VEGF) levels. ${ }^{57-59}$ In a cohort of 96 patients with cutaneous skin findings, flushing was reported in $17(16 \%)$ patients $^{58}$ and is recurrent, located on the face, trunk and abdomen with a violaceous appearance. ${ }^{60}$ 


\section{Diagnosis and Treatment}

Diagnosis relies on identifying the abnormal proliferation of plasma cells in an osteosclerotic marrow biopsy specimen and elevated monoclonal immunoglobulin in the serum or urine along with 2 of 3 major criteria (Castleman's disease, sclerotic bone lesions, elevated endothelial growth factor) and at least one minor criteria (organomegaly, volume overload, endocrinopathy, skin changes, papilledema or thrombocytosis/ polycythemia. ${ }^{57}$ Treatment is based on the extent of disease with modalities including radiation, corticosteroids and chemotherapy. ${ }^{57}$

\section{Conditions Involving Mast Cells}

Mast cell activation disorders (MCAD's) are a group of heterogeneous polygenic diseases marked by abnormal release of mast cell mediators and in some cases by the expansion and accumulation of abnormal dysfunctional mast cells in one or more organ systems (Table 3). ${ }^{61,62}$ Disorders involving the mast cell include both cutaneous and/or systemic presentations and fall into three broad categories: (1) conditions that cause enhanced abnormal mast cell proliferation, (2) conditions that enhance mast cell reactivity to endogenous or exogenous factors, and (3) unknown mast cell activation factors. Diagnosis is based on a fulfilling a specific set of well-defined diagnostic criteria that include the presence of recurrent or persistent symptoms involving two or more organ systems, evidence for elevation in serum tryptase, elevated 24-hour urinary histamine metabolite (methyhistamine) or PGD2, 11- $\beta$-PGF2 $\alpha$ and improvement or resolution of symptoms in response to antimediator therapy. ${ }^{63}$ Disease entities are further subclassified based on site and extent of disease involvement, presence of morphologic, cytogenetic and immunophenotyping features and biochemical levels (Table 2). ${ }^{63}$

Conditions that Cause Abnormal Mast Cell Proliferation Systemic mastocytosis, a stem cell-derived hematologic malignancy, consists of a group of diseases characterized by aberrant proliferation of mast cells. ${ }^{62}$ These morphological and immune-phenotypical abnormal functioning cells cause disease often confined to the skin (limited cutaneous mastocytosis) and/or in association with more widespread disease involving, liver, spleen, bone marrow, lymph nodes and gastrointestinal tract. Criteria for the diagnosis of systemic mastocytosis was established and updated by the World Health Organization (WHO) in 2016 (Table 4). ${ }^{62}$

Cutaneous disease involves abnormal mast cells confined to the skin and includes maculopapular cutaneous mastocytosis (formerly known as urticarial pigmentosa), localized mastocytoma and diffuse cutaneous mastocytosis (Table 5). ${ }^{64}$ It is important to recognize that most children with disease limited to their skin do not develop systemic mastocytosis. Conversely, skin involvement generally in the form of maculopaular cutaneous mastocytosis most commonly occurs in adult patients with systemic mastocytosis, particularly isolated system mastocytosis, the most common subtype. A prevalence of systemic mastocytosis of 1 in 10,000 and incidence (including cutaneous disease) of approximately 0.89 per 100,000 years has been reported. ${ }^{65}$

Subgroups of systemic mastocytosis (SM) are classified based on extent and disease severity, bone marrow findings (eg, dysplasia or myeloproliferation of non-mast cell lines with percentage of neoplastic mast cell involvement), presence of organ system enlargement (B-findings, eg, hepatomegaly, splenomegaly, lymphadenopathy) and dysfunction (C-findings, eg, malabsorption, bone fractures and cytopenias), associated myeloid, myeloproliferative or myelodysplastic syndrome and include the following five categories (Table 2): (1) indolent systemic mastocytosis (ISM), (2) SM with associated hematologic neoplasm (SM-AHND), (3) smoldering systemic mastocytosis (SSM),

Table 3. Diagnostic Criteria for Mast Cell Activation Disorder (All three Required)

\section{Clinical signs and symptoms involving $\geq 2$ organs or systems related to recurrent or chronic systemic mast} cell activation.

Dermatology: Flushing*, dermatographism, pruritus, maculopapular cutaneous mastocytosis, angioedema.

Pulmonary: Wheezing, sore throat, stridor.

Cardiovascular: Chest pain, hypotension, tachycardia.

Gastroenterology: abdominal pain*, diarrhea*, bloating*, nausea, vomiting, malabsorption, esophagitis.

Nasal-ocular: nasal congestion, pruritus.

Neurology: Headache*, memory and concentration difficulties ${ }^{*}$, paresthesia, peripheral neuropathy

Musculoskeletal: Bone and muscle pain, degenerative disc disease, osteopenia or osteoporosis.

2. Elevated serum Tryptase level (preferably) or elevated 24-h N-methylhistamine or PGD/11 -PGF2 levels.

Tryptase level $>20 \mathrm{ng} / \mathrm{ml}$ during $\geq 2$ symptomatic episodes OR at least a single level $>15 \mathrm{ng} / \mathrm{ml}$ if the persistent baseline level above $15 \mathrm{ng} / \mathrm{ml}$.

\section{Decrease in frequency, severity, or resolution of symptoms in response to antimediator therapy.}

First and second generation histamine antagonist ( $\mathrm{H} 1$ antagonist) with or without $\mathrm{H} 2$ histamine antagonist 2 ( $\mathrm{H} 2$ antagonist), leukotriene antagonist, or 5-lipoxygensae inhibitor, or mast cell stabilizer (cromolyn sodium)

\footnotetext{
${ }^{\star}$ Common symptoms
} 
(4) aggressive systemic mastocytosis (ASM) and variant in transformation, and (5) mast cell leukemia (MCL-Acute and Chronic subtypes).

A mutation in KIT (Ki-67), most commonly involving codon $\mathrm{D} 816 \mathrm{~V}$, is responsible for enhanced neoplastic mast cell differentiation, maturation and survival due to constitutive activation of the KIT tyrosine kinase activity. ${ }^{62,65}$ Prognosis for patients with SM is highly variable and depends upon the disease subtype. Indolent disease has a better prognosis with most patients having a normal life expectancy, and aggressive disease and mast cell leukemia typically have a poor longterm prognosis. ${ }^{66}$

Conditions that Enhance Mast Cell Reactivity and Hyperplasia to Endogenous or Exogenous Factors

These MCADs include known IgE hypersensitivity and drug induced reactions, and hyperplasia of mast cells in response to chronic infection, neoplasia and autoimmune stimuli. In contrast to systemic mastocytosis, no mutation in KIT D816V or CD25 cells is present.

\section{Unknown Mast Cell Activation Factors}

An idiopathic category defines MCADs that occur in the absence of a known underlying reactive disease or abnormal mast cell proliferative disorder. It is a diagnosis of exclusion met when testing for systemic mastocytosis is negative, there is insufficient evidence to suggest mast cell clonal abnormalities, and no chronic inflammatory, neoplastic, drug-induced or known allergen-specific IgE is identified. ${ }^{63,67}$

\section{Clinical Presentation and Gastrointestinal Involvement}

A diagnosis of a MCAD should be considered in patients presenting with recurrent unexplained episodes of anaphylaxis marked by hypotension and syncope, flushing, cutaneous skin lesions, pruritus, unexplained osteoporosis and gastrointestinal symptoms including abdominal cramping, nausea and diarrhea. ${ }^{68,69}$ Flushing caused by PGD2 release is common and described as bright red, pruritic and burning, associated with gastrointestinal symptoms, hemodynamic instability and diaphoresis. ${ }^{24,63}$ Episodes typically last 15 to 30 minutes.
Factors known to trigger mast cell degranulation such as hymenoptera stings, stress, trauma, exercise, temperature extremes, general anesthesia, and use of non-steroidal antiinflammatory medications, alcohol, narcotic, certain antimicrobials, muscle relaxants and iodinated radiocontrast should be sought from the history. The clinical presentation of MCADs is based on the predominant organ(s) involved and specific mediators released.

Symptoms of flushing with diarrhea and abdominal cramping may resemble other clinical syndromes including carcinoid syndrome, pheochromocytoma and medullary thyroid carcinoma. $^{70}$ Gastric acid hypersecretion, altered bowel motility and malabsorption caused by bowel infiltration, injury and edema are believed to be involved in diarrhea. A number of mechanisms have been postulated to explain the diarrhea including gastric hypersecretion, intestinal villi destruction by increased acidity and prostaglandin D2 oversynthesis causing malabsorption. ${ }^{71}$

Gastrointestinal tract symptoms (nausea, vomiting, and abdominal pain) are frequently observed and occur based on the location and density of the mast cell infiltration. The most frequent symptoms are bloating and diarrhea followed by abdominal pain and nausea. ${ }^{24,72-74}$ Symptoms may be caused by peptic ulcer disease, esophagitis gastrointestinal edema or dysmotility.

The pathogenesis for the abdominal pain seen in systemic mastocytosis involves infiltration of mast cell within the intestines, liver and spleen and/or release of mediators including histamine and prostaglandin D2. ${ }^{24}$ Other local gastrointestinal peptides causing diarrhea and abdominal pain include VIP, neurotensin, substance P or motilin and gastrin.74

Hepatosplemomegaly with or without liver and splenic dysfunction and lymphadenopathy may be present in some patients with systemic mastocytosis (B and C-findings). Elevated levels of liver alkaline phosphatase and v-glutamyl transpeptidase are found in patients with severe infiltrative hepatic disease, which may lead to portal hypertension and ascites. ${ }^{75}$ Endoscopic and/or colonoscopic biopsies should be

Table 4. Diagnostic Criteria for System Mastocytosis 2016 WHO Classification (Requires 1 major and 1 minor or 3 minor criteria)

\section{Major Criterion}

Multifocal dense infiltrate of mast cells ( $\geq 15$ in aggregate) in bone marrow biopsies and/or in sections of other extracutaneous organ(s)

\section{Minor Criterion}

$>25 \%$ of all mast cells are atypical (type I or type II) on bone marrow smear or are spindle-shaped in infiltrates detected on sections of visceral organs

KIT point mutation at codon 816 in the bone marrow or another extracutaneous organ

Mast cells in bone marrow or blood or another extracutaneous organ exhibit CD2 and/or CD25

Baseline serum tryptase level $>20 \mathrm{ng} / \mathrm{ml}$ (in case of unrelated myeloid neoplasm, this item is not a valid criterion 
Table 5. Diagnostic Criteria from EU-Consensus Group for Mastocytosis with Cutaneous Disease confined to the skin. [Mastocytosis in Skin (MIT)] ${ }^{\star \star}$

\section{Major}

Typical skin lesions of mastocytosis associated with Darier's Sign

Minor

Increased numbers of mast cell in biopsy of skin lesion.

Activating KIT mutation detected in skin lesion.

${ }^{* *}$ Confirmed when major and one minor criteria is fulfilled OR if Darier's sign absent or slightly positive than diagnosis made based on meeting both Minor Criteria.

considered in patients with systemic mastocytosis presenting with intractable gastrointestinal symptoms to exclude other pathological conditions. ${ }^{73}$

\section{Diagnosis and Treatment}

Bone marrow aspiration and biopsy are important tools in diagnosing mastocytosis in adults. Flow cytometry and polymerase chain reaction (PCR) of a bone marrow aspirate sample is useful in analyzing for the KIT gene mutation and mast cell markers in patient with suspected systemic mastocytosis. ${ }^{65}$

Biochemical confirmation of mast cell degranulation includes identifying an elevated serum tryptase levels measured between 15 minutes and 4 hours after the episode. Objective significant confirmation requires that the tryptase value should be at least $20 \%$ plus $2 \mathrm{ng} / \mathrm{ml}$ above the baseline, which is typically measured at least 24 hours after symptom resolution. ${ }^{62,65}$ Serum tryptase and 24-urinary for $11-\beta$-prostaglandin F2 $\alpha$ (11- $\beta$-PGF2 $\alpha)$ further improves diagnostic accuracy for MCAS. ${ }^{76}$

Endoscopic findings in patients with systemic mastocytosis include dilated loop of small bowel with thickened, edematous or scalloped folds. Urticarial, small mucosal nodular and polypoid lesion, purple-pigmented lesion, focal areas of edema, diffuse intestinal telangiectasias lesions have been identified on colonoscopy. ${ }^{24,77}$

Treatment is focused on ameliorating symptoms. Histamine (H1 and $\mathrm{H} 2)$ receptor antagonists are first-line effective treatments for managing flushing and vasodilatory symptoms. ${ }^{66}$ Proton pump inhibitors, mast cell stabilizers, leukotriene antagonists and corticosteroids have also been used. Cromolyn sodium, a mast cell stabilizer, has been shown to be particularly effective in managing gastrointestinal symptoms. ${ }^{66}$ Leukotriene antagonists are used in patients with elevated prostaglandin D2 levels. Aspirin has been used to treat flushing but should be cautiously administered with slow, titrating, dose escalation since hypersensitivity has been reported. Glucocorticoids are effective particularly for patients with ascites and diarrhea associated with malabsorption. Epinephrine is used for treating anaphylaxis in the setting of an acute hypotensive episode.
Cytoreductive therapies comprised of interferon-alpha and/or prednisone, cladribine, tyrosine kinase inhibitors and hydroxyurea, are mainstay to reduce mast cell burden particularly for aggressive, advanced (target organ damage or C-findings) or treatment resistant systemic mastocytosis. Interferon $\alpha$ with or without prednisone is usually the first-line treatment. ${ }^{66}$ Tyrosine kinase inhibitors (imatinib, dasatinib) are used as second or third line therapy if the disease is not responsive to interferon- $\alpha$ and/or prednisone and cladribine. ${ }^{66}$

\section{Hyperbasophila}

Recent classification proposed the use of the term hyperbasophila (HB) to describe the presence of persistent peripheral basophilc counts greater than 1000 per $\mu$ lof blood. Further diagnostic evaluation is required to investigate for an underlying myeloid malignancy. ${ }^{78}$ Several secondary variant cases of Philadelphia-positive chronic myelogenous leukemia with basophilia with elevated histamine levels have been reported. ${ }^{78}$ Basophilic leukemias are rare acute or chronic malignancies that present in either acute or chronic form with primary or secondary variants.

\section{Clinical Presentation with Gastrointestinal Involvement}

Symptoms are similar to other conditions that result in excessive histamine release including flushing, pruritus and hypotension. ${ }^{79}$

\section{Diagnosis and Treatment}

Assessment involves distinguishing between transient or reactive and chronic or persistent $\mathrm{HB}$. When persisting beyond 8 weeks, HB is most likely caused by a myeloid neoplasm especially when other abnormalities in the blood cell count are identified. Initial evaluation should include a history inquiring about occupational and environmental exposures, and examination for spleen or lymph node enlargement. Blood samples for tryptase, JAK2 V617F and BCR-ABL1 should be obtained. Additional testing for expression of FIPL1-PDGFRA fusion gene is obtained in cases with concomitant eosinophilia. A bone marrow biopsy and immunohistochemistical analysis should be performed in patients where no BCR-ABL1 or other cause is found. Molecular studies and chromosomal analyses are indicated in cases of myelodysplastic syndrome and/or myeloproliferative neoplasms. ${ }^{78}$ Chronic infections, chronic autoimmune diseases or uncontrolled atopic disorders should be considered in the 
differential diagnosis in cases where no neoplasm is detected during bone marrow examination. ${ }^{78}$

Patients with basophilic leukemia who are candidates should undergo stem cell transplantation. In patients who are not transplant candidates, targeted drug therapy, polychemotherapy or palliative cytoreductive treatments are administered. ${ }^{78} \mathrm{H} 1$ and $\mathrm{H} 2$ receptor antagonist should be administered prophylactically in all patients.

\section{Conclusion}

The differential diagnosis of flushing can be challenging, since it encompasses both benign and malignant conditions and includes a wide variety of overlapping, and in most cases, non-specific gastrointestinal symptoms. Initial evaluation involves obtaining a detailed history and physical examination inquiring about the frequency and duration of the attacks, description and location of the flush and precipitating factor(s). The constellation of flushing, diarrhea and hypotension suggests mast cell activation syndromes, anaphylaxis, pheochromocytomas, scromboid poisoning, and carcinoid syndrome. Other conditions to consider that present acutely and generally caused by a precipitating factor include scromboid poisoning and anaphylaxis. The absence of sweating with pruritus and burning pain with flushing may further be helpful in narrowing the differential diagnosis of a mast cell activation disorder.

Screening and obtaining specific diagnostic laboratory and radiologic studies is guided based on recognizing the clinical patterns and associated symptoms of disease. Radiologic imaging should be reserved and performed after initial screening biochemical tests suggest potential etiologies. Diagnosis in some cases may require tissue biopsy and cytohistochemical analysis.

\section{Acknowledgements}

The authors would like to thank Dr. Hale Toklu and Ms. Megan Hubbard for their contributions.

\section{References}

1. Hannah-Shmouni F, Stratakis CA, Koch CA. Flushing in (neuro) endocrinology. Rev Endocr Metab Disord. 2016;17(3):373380.

2. Izikson L, English JC III, Zirwas MJ. The flushing patient: Differential diagnosis, workup, and treatment. J Am Acad Dermatol. 2006;55(2):193-208.

3. Wilkin JK. Why is flushing limited to a mostly facial cutaneous distribution? J Am Acad Dermatol. 1988;19(2):309-313.

4. Wilkin JK. The red face: Flushing disorders. Clin Dermatol. 1993;11(2):211-223.

5. Surawicz CM. Mechanisms of Diarrhea. Curr Gastroenterol Rep. 2010;12(4):236-241

6. Maton PN. The carcinoid syndrome. JAMA. 1988;260(11):16021605.

7. Singh S, Asa SL, Dey C, et al. Diagnosis and management of gastrointestinal neuroendocrine tumors: An evidence-based Canadian consensus. Cancer Treat Rev. 2016;47:32-45.
8. Klöppel G, Rindi G, Anlauf M, Perren A, Komminoth P. Sitespecific biology and pathology of gastroenteropancreatic neuroendocrine tumors. Virchows Arch. 2007;451(S1)(Suppl 1):9-27.

9. Burke AP, Thomas RM, Elsayed AM, Sobin LH. Carcinoids of the jejunum and ileum: an immunohistochemical and clinicopathologic study of 167 cases. Cancer 1997;79(6):1086-1093.

10. Pan SY, Morrison H. Epidemiology of cancer of the small intestine. World J Gastrointest Oncol 2011;3(3):33-42.

11. Griniatsos J, Michail O. Appendiceal neuroendocrine tumors: Recent insights and clinical implications. World Journal of Gastrointestinal Oncology. 2010;2(4):192-196.

12. Yao JC, Hassan M, Phan A, et al. One hundred years after carcinoid: epidemiology of and prognostic factors for neuroendocrine tumors in 35,825 cases in the United States. J Clin Oncol. 2008;26(18):3063-3072.

13. Hirabayashi K, Zamboni G, Nishi T, Tanaka A, Kajiwara H, Nakamura N. Histopathology of gastrointestinal neuroendocrine neoplasms. Frontiers in Oncology. 2013;3:2.

14. ASCO Cancer.Net. Carcinoid Tumor: Statistics. 2016

15. Ramage JK, Ahmed A, Ardill J, et al; UK and Ireland Neuroendocrine Tumour Society. Guidelines for the management of gastroenteropancreatic neuroendocrine (including carcinoid) tumours (NETs). Gut. 2012;61(1):6-32.

16. Shebani KO, Souba WW, Finkelstein DM, et al. Prognosis and survival in patients with gastrointestinal tract carcinoid tumors. Ann Surg 1999;229(6):815-821; discussion 822-813.

17. Eriksson B, Öberg K, Stridsberg M. Tumor markers in neuroendocrine tumors. Digestion. 2000;62(1):33-38.

18. Mohyi D, Tabassi K, Simon J. Differential diagnosis of hot flashes. Maturitas. 1997;27(3):203-214.

19. El Tal AK, Tannous Z. Cutaneous vascular disorders associated with internal malignancy. Dermatol Clin. 2008;26(1):45-57, viii.

20. Anthony LB, Strosberg JR, Klimstra DS, et al; North American Neuroendocrine Tumor Society (NANETS). The NANETS consensus guidelines for the diagnosis and management of gastrointestinal neuroendocrine tumors (nets): welldifferentiated nets of the distal colon and rectum. Pancreas 2010;39(6):767-774.

21. Tomassetti P, Migliori M, Lalli S, Campana D, Tomassetti V, Corinaldesi R. Epidemiology, clinical features and diagnosis of gastroenteropancreatic endocrine tumours. Ann Oncol. 2001;12(suppl 2):S95-S99.

22. Ohe MR, Camilleri M, Kvols LK, Thomforde GM. Motor dysfunction of the small bowel and colon in patients with the carcinoid syndrome and diarrhea. N Engl J Med. 1993;329(15):1073-1078.

23. Ghevariya V, Malieckal A, Ghevariya N, Mazumder M, Anand S. Carcinoid tumors of the gastrointestinal tract. South Med J. 2009;102(10):1032-1040.

24. Lee JK, Whittaker SJ, Enns RA, Zetler P. Gastrointestinal manifestations of systemic mastocytosis. World J Gastroenterol. 2008;14(45):7005-7008.

25. Liu EH, Solorzano CC, Katznelson L, Vinik AI, Wong R, Randolph G. AACE/ACE disease state clinical review: diagnosis and management of midgut carcinoids. Endocr Pract. 2015;21(5):534-545.

26. Eckhauser FE, Argenta LC, Strodel WE, et al. Mesenteric angiopathy, intestinal gangrene, and midgut carcinoids. Surgery. 1981;90(4):720-728.

27. Modlin IM, Shapiro MD, Kidd M. Carcinoid tumors and fibrosis: an association with no explanation. Am J Gastroenterol. 2004;99(12):2466-2478.

28. Strobbe L, DHondt E, Ramboer C, Ceuppens H, Hinnekens P, Verhamme M. Ileal carcinoid tumors and intestinal ischemia. Hepatogastroenterology. 1994;41(5):499-502. 
29. Öhrvall U, Eriksson B, Juhlin C, et al. Method for dissection of mesenteric metastases in mid-gut carcinoid tumors. World $\mathrm{J}$ Surg. 2000;24(11):1402-1408.

30. Warner TF, O'Reilly G, Lee GA. Mesenteric occlusive lesion and ileal carcinoids. Cancer 1979;44(2):758-762.

31. Druce M, Rockall A, Grossman AB. Fibrosis and carcinoid syndrome: from causation to future therapy. Nat Rev Endocrinol. 2009;5(5):276-283.

32. Kidd M, Modlin IM, Pfragner R, et al. Small bowel carcinoid (enterochromaffin cell) neoplasia exhibits transforming growth factor- $\beta 1$-mediated regulatory abnormalities including up-regulation of C-Myc and MTA1. Cancer. 2007;109(12):2420-2431.

33. Phan AT, Öberg K, Choi J, et al; North American Neuroendocrine Tumor Society (NANETS). NANETS consensus guideline for the diagnosis and management of neuroendocrine tumors: well-differentiated neuroendocrine tumors of the thorax (includes lung and thymus). Pancreas 2010;39(6):784-798.

34. Bendelow J, Apps E, Jones LE, Poston GJ. Carcinoid syndrome. European Journal of Surgical Oncology (EJSO). 2008;34(3):289-296.

35. Beaumont JL, Cella D, Phan AT, Choi S, Liu Z, Yao JC. Comparison of health-related quality of life in patients with neuroendocrine tumors with quality of life in the general US population. Pancreas. 2012;41(3):461-466.

36. Caplin ME, Pavel M, Ruszniewski P. Lanreotide in metastatic enteropancreatic neuroendocrine tumors. N Engl J Med. 2014;371(16):1556-1557.

37. Rinke A, Müller HH, Schade-Brittinger C, et al; PROMID Study Group. Placebo-controlled, double-blind, prospective, randomized study on the effect of octreotide LAR in the control of tumor growth in patients with metastatic neuroendocrine midgut tumors: a report from the PROMID Study Group. J Clin Oncol. 2009;27(28):4656-4663.

38. Zileli M, Kalayci M, Başdemir G. Paraganglioma of the thoracic spine. J Clin Neurosci. 2008;15(7):823-827.

39. Reisch N, Peczkowska M, Januszewicz A, Neumann HPH. Pheochromocytoma: presentation, diagnosis and treatment. J Hypertens. 2006;24(12):2331-2339.

40. Feng N, Zhang WY, Wu XT. Clinicopathological analysis of paraganglioma with literature review. World J Gastroenterol. 2009;15(24):3003-3008.

41. Lenders JWM, Duh QY, Eisenhofer G, et al; Endocrine Society. Pheochromocytoma and paraganglioma: an endocrine society clinical practice guideline. J Clin Endocrinol Metab. 2014;99(6):1915-1942.

42. Vázquez-Quintana E, Vargas R, Pérez M, et al. Pheocromocytoma and gastrointestinal bleeding. Am Surg. 1995;61(11):937-939.

43. Waguespack SG, Rich T, Grubbs E, et al. A current review of the etiology, diagnosis, and treatment of pediatric pheochromocytoma and paraganglioma. J Clin Endocrinol Metab. 2010;95(5):2023-2037.

44. Mouri T, Takahashi K, Sone M, et al. Calcitonin gene-related peptide-like immunoreactivities in pheochromocytomas. Peptides. 1989;10(1):201-204.

45. Nicholls MG, Lainchbury JG, Lewis LK, et al. Bioactivity of adrenomedullin and proadrenomedullin N-terminal 20 peptide in man. Peptides. 2001;22(11):1745-1752.

46. Ikuta S, Yasui C, Kawanaka M, et al. Watery diarrhea, hypokalemia and achlorhydria syndrome due to an adrenal pheochromocytoma. World J Gastroenterol. 2007;13(34):4649-4652.

47. Fishbein L. Pheochromocytoma and paraganglioma: genetics, diagnosis, and treatment. Hematol Oncol Clin North Am. 2016;30(1):135-150.
48. Lam S, Liew H, Khor HT, et al. VIPoma in a 37-year-old man. Lancet. 2013;382(9894):832.

49. Vasoactive Intestinal Peptide Tumor (VIPoma). In: De Groot LJ, Chrousos G, Dungan K, et al, ed. Endotext [Internet]. South Dartmouth (MA): MDText.com, Inc.; 2013.

50. Feurle GE, Helmstaedter V, Tischbirek K, et al. A multihormonal tumor of the pancreas producing neurotensin. Dig Dis Sci 1981;26(12):1125-1133.

51. Shulkes A, Boden R, Cook I, Gallagher N, Furness JB. Characterization of a pancreatic tumor containing vasoactive intestinal peptide, neurotensin, and pancreatic polypeptide. J Clin Endocrinol Metab. 1984;58(1):41-48.

52. Kyriacou A, McLaughlin J, Syed AA. Thyroid disorders and gastrointestinal and liver dysfunction: A state of the art review. Eur J Intern Med. 2015;26(8):563-571.

53. Kebebew E, Ituarte PHG, Siperstein AE, et al. Medullary thyroid carcinoma: clinical characteristics, treatment, prognostic factors, and a comparison of staging systems. Cancer 2000;88(5):1139-1148.

54. Beressi N, Campos JM, Beressi JP, et al. Sporadic medullary microcarcinoma of the thyroid: a retrospective analysis of eighty cases. Thyroid. 1998;8(11):1039-1044.

55. Lupoli GA, Fonderico F, Fittipaldi MR, et al. The role of somatostatin analogs in the management of medullary thyroid carcinoma. J Endocrinol Invest. 2003;26(8)(Suppl):72-74.

56. Dispenzieri A. POEMS syndrome: Update on diagnosis, riskstratification, and management. Am J Hematol. 2015;90(10):951-962.

57. Dispenzieri A, Buadi FK. A review of POEMS syndrome. Oncology (Williston Park). 2013;27(12):1242-1250.

58. Miest RYN, Comfere NI, Dispenzieri A, Lohse CM, el-Azhary RA. Cutaneous manifestations in patients with POEMS syndrome. Int J Dermatol. 2013;52(11):1349-1356.

59. Thijs AMJ, van Herpen CML, Sweep FCGJ, et al. Role of endogenous vascular endothelial growth factor in endothelium-dependent vasodilation in humans. Hypertension. 2013;61(5):1060-1065.

60. Myers BM, Miralles GD, Taylor CA, Gastineau DA, Pisani RJ, Talley NJ. Poems syndrome with idiopathic flushing mimicking carcinoid syndrome. Am J Med. 1991;90(5):646648.

61. Petra AI, Panagiotidou S, Stewart JM, Conti P, Theoharides TC. Spectrum of mast cell activation disorders. Expert Rev Clin Immunol. 2014;10(6):729-739.

62. Valent P, Akin C, Metcalfe DD. Mastocytosis: 2016 updated WHO classification and novel emerging treatment concepts. Blood. 2017;129(11):1420-1427.

63. Afrin LB, Pöhlau D, Raithel M, et al. Mast cell activation disease: An underappreciated cause of neurologic and psychiatric symptoms and diseases. Brain Behav Immun. 2015;50:314-321.

64. Hartmann K, Escribano L, Grattan C, et al. Cutaneous manifestations in patients with mastocytosis: Consensus report of the European Competence Network on Mastocytosis; the American Academy of Allergy, Asthma \& Immunology; and the European Academy of Allergology and Clinical Immunology. J Allergy Clin Immunol. 2016;137(1):35-45.

65. Ustun C, Arock M, Kluin-Nelemans HC, et al. Advanced systemic mastocytosis: from molecular and genetic progress to clinical practice. Haematologica. 2016;101(10):1133-1143.

66. Pardanani A. Systemic mastocytosis in adults: 2015 update on diagnosis, risk stratification, and management. Am J Hematol. 2015;90(3):250-262.

67. Akin C, Valent P, Metcalfe DD. Mast cell activation syndrome: proposed diagnostic criteria. J Allergy Clin Immunol 2010;126(6):1099-1104.e4. 
68. Hamilton MJ, Hornick JL, Akin C, et al. Mast cell activation syndrome: a newly recognized disorder with systemic clinical manifestations. J Allergy Clin Immunol 2011;128(1):147-152. e2.

69. Smith JH, Butterfield JH, Pardanani A, DeLuca GC, Cutrer FM. Neurologic symptoms and diagnosis in adults with mast cell disease. Clin Neurol Neurosurg. 2011;113(7):570-574.

70. Picard M, Giavina-Bianchi P, Mezzano V, Castells M. Expanding spectrum of mast cell activation disorders: monoclonal and idiopathic mast cell activation syndromes. Clin Ther. 2013;35(5):548-562.

71. Sokol H, Georgin-Lavialle S, Grandpeix-Guyodo C, et al. Gastrointestinal involvement and manifestations in systemic mastocytosis. Inflamm Bowel Dis. 2010;16(7):1247-1253.

72. Bains SN, Hsieh FH. Current approaches to the diagnosis and treatment of systemic mastocytosis. Ann Allergy Asthma Immunol. 2010;104(1):1-10; quiz 10-12, 41.

73. Behdad A, Owens SR. Systemic mastocytosis involving the gastrointestinal tract: case report and review. Arch Pathol Lab Med. 2013;137(9):1220-1223.

74. Cherner JA, Jensen RT, Dubois A, et al. Gastrointestinal dysfunction in systemic mastocytosis. A prospective study. Gastroenterology 1988;95(3):657-667.

75. Carter MC, Metcalfe DD, Komarow HD. Mastocytosis. Immunol Allergy Clin North Am. 2014;34(1):181-196.

76. Ravi A, Butterfield J, Weiler CR. Mast cell activation syndrome: improved identification by combined determinations of serum tryptase and 24-hour urine $11 \beta$-prostaglandin $2 \alpha$. The Journal of Allergy and Clinical Immunology: In Practice. 2014;2(6):775-778.

77. Scolapio JS, Wolfe J III, Malavet P, Woodward TA. Endoscopic findings in systemic mastocytosis. Gastrointest Endosc. 1996;44(5):608-610.

78. Valent P, Sotlar K, Blatt K, et al. Proposed diagnostic criteria and classification of basophilic leukemias and related disorders. Leukemia. 2017;31(4):788-797.

79. Greil R, Pleyer L, Neureiter D, Faber V, eds. Chronic myeloid neoplasias and clonal overlap syndromes: epidemiology, pathophysiology, and treatment options. New York: SpringerVerlag Wien; 2010.

\section{Author Affiliations}

Vaibhav Rastogi, MD*, $;$; Devina Singh, MDf; Joseph J. Mazza, MD ; Dipendra Parajuli, MDף, Steven H. Yale, $M D^{*}$,

*University of Central Florida College of Medicine/HCA Consortium Graduate Medical Education, North Florida Regional Medical Center, $6500 \mathrm{~W}$ Newberry Rd, Gainesville, FL 32605.

†University of Central Florida College of Medicine, 6850

Lake Nona Blvd, Orlando, FL 32827.

$\$$ Feinstein Institute for Medical Research, 350 Community

Dr. Manhasset, NY 11030

\$Marshfield Clinic Research Institute, 1000 North Oak

Avenue, Marshfield, WI 54449.

ๆUniversity of Louisville, Department of Medicine,

Gastroenterology, Hepatology and Nutrition. Director,

Fellowship Training Program, Director, Medical Procedure

Unit Louisville VAMC 401 East Chestnut Street, Louisville,

KY 40202. 